\title{
NATURAL CONVECTION ON HEAT TRANSFER FLOW OF NON- NEWTONIAN SECOND GRADE FLUID OVER HORIZONTAL CIRCULAR CYLINDER WITH THERMAL RADIATION
}

\section{Ramachandrprasad ${ }^{1}$, R. Bhuvanavijaya ${ }^{2}$ and B. Mallikarjuna ${ }^{3 *}$}

${ }^{1}$ Department of Mathematics, Madanapalle Institute of Technology and Science, Madanapalle, India-517325, India, Email: rcpmaths@gmail.com

${ }^{2}$ Department of Mathematics, JNT University College of Engineering (Autonomous) Anantapur, Ananthapuramu, AndhraPradesh -515002, India, Email: bhuvanarachamalla@gmail.com

${ }^{3}$ Department of Mathematics, BMS College of Engineering, Bangalore, Karnataka-560019, India,

Email:mallikarjuna.jntua@gmail.com, mallikarjunab.maths@bmsce.ac.in

* Corresponding Author

\begin{abstract}
:
This article numerically studies for multi-physical transport of an optically-dense, free convective incompressible non-Newtonian second grade fluid past an isothermal, impermeable horizontal circular cylinder. The governing boundary layer equations for momentum and energy transport, which are parabolic in nature, have been reduced to non-similarity non-linear conservation equations using appropriate transformations and then solved numerically by employing with most validated, efficient implicit finite difference method with Keller box scheme. The numerical code is validated with previously existing results and found to be very good agreement. Numerical results have been carried out for various values of the physical parameters; Deborah number $(0 \leq D e \leq 1.5)$, Prandtl number $(0 \leq \operatorname{Pr} \leq 100)$ and thermal radiation $(0 \leq R \leq 5)$ on flow velocity and temperature profiles. Furthermore, the effects of these parameters on non-dimensional wall shear stress (skin friction) and surface heat transfer rate (Nusselt number) are also investigated. Increasing the Deborah number reduces velocity profile, skin friction where as it enhances the temperature profile. Increasing Prandtl number decelerates the flow velocity, temperature and skin friction. Increase in radiation parameter retards the flow velocity, temperature profiles and skin friction. The rate of heat transfer (Nusselt number) enhances markedly with increase in radiation parameter and Prandtl number but depreciated for larger values of Deborah number. Increasing stream wise coordinate retards the velocity gradient whereas enhances rate of heat transfer. Applications of the model arise in polymer processing in chemical engineering, metallurgical material processing.
\end{abstract}

Keywords: Non-Newtonian fluid; Second Grade fluid; Thermal Radiation; Prandtl number; Skin friction; Nusselt Number; Keller Box Scheme

\section{NOMENCLATURE}

$\mathrm{u} \quad$ Velocity component in $\mathrm{x}$-direction $[\mathrm{m} / \mathrm{s}]$

V Velocity component in y-direction $[\mathrm{m} / \mathrm{s}]$

g Acceleration due to gravity $\left[\mathrm{m} / \mathrm{s}^{2}\right]$

f Non-dimensional stream function

a Radius of the cylinder [m]

$\mathrm{T}_{\mathrm{w}} \quad$ Surface temperature $[\mathrm{K}]$

$\mathrm{T}_{\infty} \quad$ Ambient fluid temperature $[\mathrm{K}]$

$\mathrm{x} \quad$ stream wise coordinate [m]

y Transverse coordinate [m]

$\mathrm{C}_{\mathrm{f}} \quad$ Skin friction coefficient

$\mathrm{Nu} \quad$ Nusselt number

\section{Greek symbols}

$\alpha_{1}, \alpha_{2} \quad$ Material fluid parameters

$\rho \quad$ Fluid density $\left[\mathrm{kg} / \mathrm{m}^{3}\right]$

$\xi \quad$ The dimensionless tangential coordinate

$\eta \quad$ The dimensionless radial coordinate

$\beta \quad$ Thermal expansion coefficient $[1 / \mathrm{K}]$

$\sigma \quad$ Stefan-Bolzmann constant

$\tau_{x x} \quad$ Normal stress in $\mathrm{x}$-direction

$\tau_{y y} \quad$ Normal stress in y-direction

$\tau_{x y}, \tau_{y x} \quad$ Shear stresses

$\psi \quad$ Dimensionless Stream function

$\theta \quad$ Non-dimensional temperature 


$\begin{array}{ll}G r & \text { Grashof number } \\ \mathrm{De} & \text { Deborah number } \\ \mathrm{A}_{1}, \mathrm{~A}_{2} & \text { Rivlin-Ericksen tensors } \\ k & \begin{array}{l}\text { Thermal conductivity of the second grade } \\ \text { fluid }\left\lceil\mathrm{m}^{2} / \mathrm{s}\right\rceil\end{array}\end{array}$

\section{Introduction}

The study of flow and heat transfer generated by different geometries in non-Newtonian fluid plays a significant role in many engineering and industrial areas. Examples of such fluids are foods, fossils, fuels, pulps and molten polymers, several fluids in pharmaceutical formulations, synthetic lubricants, salvia, synovial fluid, jams, marmalades, sewage sludge, clay and coal in water and paints. During the last five years, the boundary layer flows of non-Newtonian fluids over different geometries with the influence of thermal radiation have been studied. This is in view of much importance in industrial, engineering, geo-physics and bio-science such as slurries, suspensions, dispersions, pharmaceutical formulations, paints, biological fluids, synthetic polymers, melts and solutions of naturally occurring high molecular weight, synthetic lubricants and food stuffs, naturally occurring fluids such as animal blood, behavior of exotic lubricants, liquid crystals and colloidal suspensions. Tai and Char (2010) studied the effects of Soret and Dufour on free convection flow of non-Newtonian fluids along a vertical plate embedded in a porous medium with thermal radiation. Hayat and Qasim (2011) analyzed the effects and radiation and magnetic fields on the unsteady mixed convection flow of a second grade fluid over a vertical stretching sheet. Mukhopadhyay et al (2012) investigated thermal radiation on forced convective flow and heat transfer past a permeable vertical surface in Darcy-Forchheimer porous medium. His results indicate that due to suction the skin-frinction increases while the rate of heat transfer increases due to suction. Due to thermal radiation, temperature is found to decrease. The combined effects of suction and thermal radiation can be used as means of cooling. Gireesha et al (2013) studied radiation effect on mixed convective heat and mass transfer of a dusty fluid past a stretching sheet with non-uniform heat source/sink. The radiation parameter is predicted to enhance the temperature profiles of both the fluid and dust phases for the VWT and VHF cases for larger values of radiation parameter. Rashad (2014) investigated the influence of radiation and variable viscosity on unsteady MHD flow of a rotating fluid over a stretching surface in a porous medium. Srinivasacharya et. Al (2015) studied radiation effect on heat and mass transfer flow of a viscous fluid over a vertical wavy surface in a porous medium with variable properties and reported results that increasing radiation parameter tends to increase flow velocity and temperature profiles whereas concentration profile is reduced. Rashad et.al (2016) investigated thermal radiation effects on convective boundary layer flow over a rotating cone in porous medium with thermophoresis.

Previous studies indicate that not much has been presented yet regarding the flow over horizontal cylinder. Free convection around heated, horizontal cylinder for various fluids is of great importance due to its extensive industrial process and geological formulations applications. Such as the coating of wires or polymer-fiber spinning, in the assessment of aquifers, in the exploration and thermal recovery of oil, underground nuclear waste storage sites and geothermal reservoirs. Vasu et.al (2010) studied computational analysis of free convection heat and mass transfer from an isothermal horizontal circular cylinder in a micropolar fluid with Soret/Dufour effects. They reported the results that with increasing Soret number the rate of heat transfer is boosted considerably, with the converse response with increase in Dufour number. Hussain and Hussain (2010) numerically investigated natural convection in a uniformly heated circular cylinder embedded in square enclosure filled with air at different vertical locations. Nadeem et.al (2011) investigated boundary layer flow of Second grade fluid in a cylinder with heat transfer. Hussain and Hussain (2011) studied natural convection heat transfer flow in a differentially heated square enclosure with a heat generating-conducting circular cylinder at different diagonal locations. Hussain (2013) analyzed natural convection in a parallelogrammic cavity with a hot concentric circular cylinder moving at different vertical locations. Prasad et.al (2013) studied flow and heat transfer of Casson fluid from a horizontal circular cylinder with partial slip in non-Darcy porous medium. They reported that velocity increases for increasing values of Casson fluid parameter and is found to reduce the temperature profile. Tham et al (2014) studied mixed convective heat transfer flow of a nanofluid from a horizontal circular cylinder in a porous medium using Buongiorno-Darcy model. Prasenjit et al (2015) investigated unsteady mixed convection over circular cylinder in the presence of nanofluid. They found and reported results that mixed convection heat transfer of water based nanofluid can be analyzed correctly by both the artificial neural network and Gene expression programming (GEP), but GEP is found more efficient. 
To the best of our knowledge, no one has studied the influence of thermal radiation on convective heat transfer flow of a non-Newtonian second grade fluid over a horizontal circular cylinder. The governing boundary layer equations are transformed into non-dimensional equations by using non-similarity transformation and then found the numerical solutions by employing implicit finite difference method with Keller box scheme. The numerical results are reported graphically and in tabular form for various physical parameters; Deborah number, Prandtl number and thermal radiation on flow velocity and temperature profile, as well as skin friction and Nusselt number. The obtained results are validated by comparing the results with previously published work. Excellent agreement has been obtained.

\section{Mathematical Formulation}

We consider the steady, incompressible, laminar free convective flow of non-Newtonian second grade fluid over an impermeable isothermal horizontal cylinder. Fig. 1 shows the configuration of the system and co-ordinate system. Where $a$ is the radius of the cylinder. The cylinder is heated to temperature of $T_{w}$ which is surrounded by the free stream temperature $T_{\infty}$, far away from the surface. The fluid is assumed to be a gray, absorbingemitting radiation but non-scattering medium.

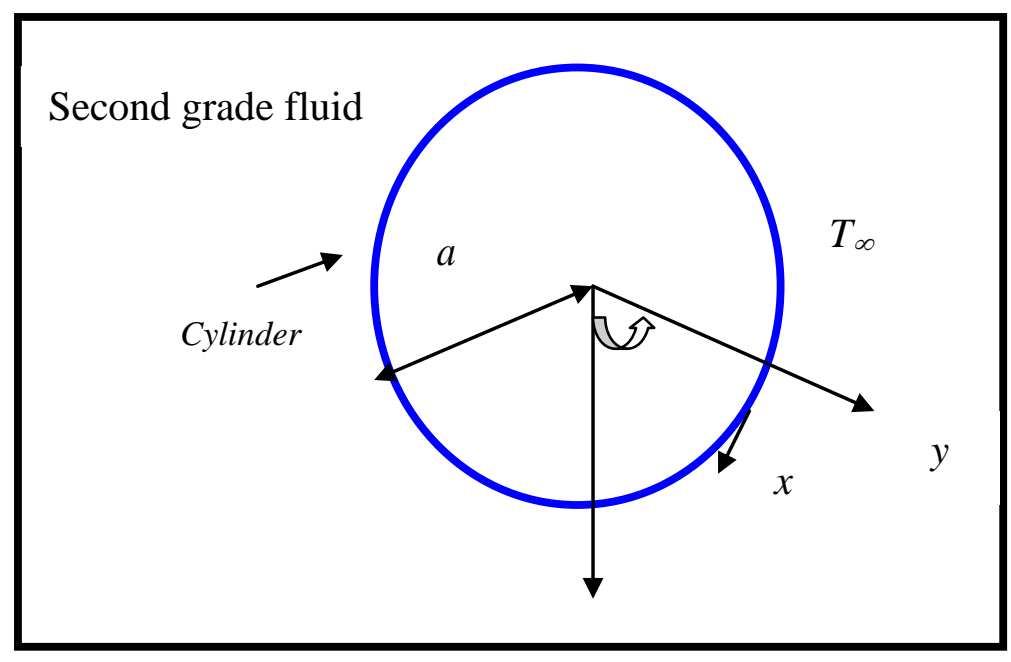

Fig. 1: Physical configuration and coordinate system

The constitutive equation for the Cauchy stress tensor related to the deformation field in a second grade fluid is $\Gamma=-p I+\mu A_{1}+\alpha_{1} A_{2}+\alpha_{2} A_{1}^{2}$

where $-p I$ is indeterminate spherical stress due to constraint of incompressibility, $\alpha_{1}, \alpha_{2}$ are the material moduli, and $A_{1}, A_{2}$ are the first two Rivlin-Ericksen (1995) tensors such that

$A_{1}=L+L^{T}, A_{2}=\frac{D A_{1}}{D t}+A_{1} L+L^{T} A_{1}$

where $L=\nabla V, \mathrm{D} / \mathrm{Dt}$ is the material derivative, Fosdick and Rajagopal (1979) argue that the second grade fluid, modelled by Eq. (1), is to be thermodynamically compatible, in the sense that all motions of the fluid meet the Clausius - Duhem inequality together with Helmoltz free energy being at its minimum whenever the fluid is locally at rest. The thermodynamical constrains put some restriction on the sign and magnitude of the material moduli:

$\mu \geq 0, \alpha_{1} \geq 0, \alpha_{1}+\alpha_{2}=0$.

where $\mathrm{V}$ is the velocity field in two-dimensional i.e. $V=[u(x, y), v(x, y)], u$ and $v$ are its components in $x$ and $y$ - directions, $\nabla V$ denotes the velocity gradient tensor. In our model we use the two-dimensional deformation rate tensor, in place of the $\mathrm{A}_{1}, \mathrm{~A}_{2}$ and write the part of the stress tensor, $\tau_{i j}$ as Beard and Walters (1964)

$\tau_{i j}=2 \alpha_{1} d_{i j}+\alpha_{2} \frac{D d_{i j}}{D t}$, where $\frac{D d_{i j}}{D t}=\frac{\partial d_{i j}}{\partial t}+L_{k i} d_{k j}+L_{k j} d_{i k}$ 
where $L_{i j}=\frac{\partial u_{i}}{\partial x_{j}}$. Now $\mathrm{x}$ and $\mathrm{y}$-momentum equations are written as:

$\rho\left(u \frac{\partial u}{\partial x}+v \frac{\partial u}{\partial y}\right)=-\frac{\partial p}{\partial x}+\frac{\partial \tau_{x x}}{\partial x}+\frac{\partial \tau_{x y}}{\partial y}$

$\rho\left(u \frac{\partial v}{\partial x}+v \frac{\partial v}{\partial y}\right)=-\frac{\partial p}{\partial y}+\frac{\partial \tau_{y x}}{\partial x}+\frac{\partial \tau_{y y}}{\partial y}$

where $\tau_{x x}, \tau_{y y}$ express the stresses in the perpendicular direction and the shear stresses are represented by $\tau_{x y}, \tau_{y x}$. Under the boundary layer approximations and Boussinesq approximation, we consider the governing equations for the mass, momentum and energy equations

$\frac{\partial u}{\partial x}+\frac{\partial v}{\partial y}=0$

$u \frac{\partial u}{\partial x}+v \frac{\partial u}{\partial y}=v \frac{\partial^{2} u}{\partial y^{2}}+\frac{\alpha_{1}}{\rho}\left[\frac{\partial}{\partial x}\left(u \frac{\partial^{2} u}{\partial y^{2}}\right)+\frac{\partial u}{\partial y} \frac{\partial^{2} v}{\partial y^{2}}+v \frac{\partial^{3} u}{\partial y^{3}}\right]+g \beta\left(T-T_{\infty}\right) \sin \left(\frac{x}{a}\right)$

$u \frac{\partial T}{\partial x}+v \frac{\partial T}{\partial y}=\alpha \frac{\partial^{2} T}{\partial y^{2}}-\frac{1}{\rho c_{p}} \frac{\partial q_{r}}{\partial y}$

The associated boundary conditions are

$u=0, v=0, T=T_{w} \quad$ at $\quad y=0$

$u=0, T=T_{\infty} \quad$ as $\quad y \rightarrow \infty$

Where $u, v$ are velocity components in $\mathrm{x}$ and $\mathrm{y}$-directions. $\alpha_{1}$ is the material constant associated with second grade fluid, $\rho$ is the fluid density, $v$ is the kinematic viscosity, $\mathrm{g}$ is the acceleration due to gravity, $\beta$ is the thermal expansion coefficient and $c_{p}$ is the specific heat at constant pressure. Second term in right hand side of Eq. (8) represents second grade fluid which consists of mixed derivates of third order derivates. Hence, momentum boundary layer equations attains higher order than the viscous (Newtonian) Navier-Stokes flow model. The impact of non-Newtonian effect takes place only on shear term of momentum equation but not on convection term. The last term on Eq. (8) represents thermal buoyancy force term which couples with temperature field of Eq. (9). The penultimate term in eq. (9) represents thermal radiative heat flux $\left(\mathrm{q}_{\mathrm{r}}\right)$, which is approximated for an optically thick boundary layer according to Rosseland approximation. The Rosseland diffusion flux model is an algebraic approximation and defined as follows:

$q_{r}=-\frac{4 \sigma}{3 k^{*}} \nabla T^{4}$

where $\sigma, k^{*}$ are respectively the Stefan-Bolzmann constant and mean absorption coefficient. The term $T^{4}$ expressed as a linear function of the temperature and it is accomplished by expanding in a Taylor series about $T_{\infty}$ and neglecting the higher order terms of $T_{\infty}$ to yield

$T^{4} \cong 4 T_{\infty}^{3} T-3 T_{\infty}^{4}$

Therefore, eq. (9) becomes

$u \frac{\partial T}{\partial x}+v \frac{\partial T}{\partial y}=\alpha \frac{\partial^{2} T}{\partial y^{2}}+\frac{16 \sigma T_{\infty}^{3}}{3 k} \frac{\partial^{2} T}{\partial y^{2}}$

In order to transform the governing equations into non-dimensional form we introduce the following nondimensional variables

$\xi=\frac{x}{a}, \quad \eta=\frac{y}{a} G r^{1 / 4}, \quad f(\xi, \eta)=\frac{\psi}{v \xi G r^{1 / 4}}, \quad \theta(\xi, \eta)=\frac{T-T_{\infty}}{T_{w}-T_{\infty}}$,

where $\psi$ is the stream function which satisfy Eq. (7) such that $(u, v)=\left(\frac{\partial \psi}{\partial y},-\frac{\partial \psi}{\partial x}\right)$. By using Eq. (14), Eqs. (8) and (13) becomes 


$$
\begin{aligned}
& f^{\prime \prime \prime}+f f^{\prime \prime}-\left(f^{\prime}\right)^{2}+D e\left[2 f f^{\prime \prime \prime}-\left(f^{\prime \prime}\right)^{2}-f f^{i v}\right]+\theta \frac{\sin (\xi)}{\xi}= \\
& \xi\left[f^{\prime} \frac{\partial f^{\prime}}{\partial \xi}-f^{\prime \prime} \frac{\partial f}{\partial \xi}+D e\left(f^{\prime \prime} \frac{\partial f^{\prime \prime}}{\partial \xi}+f^{i v} \frac{\partial f}{\partial \xi}-f^{\prime} \frac{\partial f^{\prime \prime \prime}}{\partial \xi}-f^{\prime \prime \prime} \frac{\partial f^{\prime}}{\partial \xi}\right)\right] \\
& \left(1+\frac{4}{3 R}\right) \theta^{\prime \prime}+\operatorname{Pr} f \theta^{\prime}=\operatorname{Pr} \xi\left[f^{\prime} \frac{\partial \theta}{\partial \xi}-\theta^{\prime} \frac{\partial f}{\partial \xi}\right]
\end{aligned}
$$

The corresponding boundary conditions are

$$
f^{\prime}=0, \quad f=0, \quad \theta=1 \text { at } \eta=0
$$

$f^{\prime}=0, \quad \theta=0$ as $\eta \rightarrow \infty$

where primes denotes the differentiation with respect to $\eta$, the dimensionless radial coordinate, $\xi$ is the dimensionless tangential coordinate, $D e=\frac{\alpha_{1} U_{R}}{\mu a}$ is the Deborah number, $U_{R}=\frac{v G r^{1 / 2}}{a}$ is the reference velocity, $G r=\frac{g \beta\left(T_{w}-T_{\infty}\right) a^{3}}{v^{2}}$ is the free convection (Grashof) number, $\operatorname{Pr}=\frac{v}{\alpha}$ is the Prandtl number, $\alpha=\frac{k}{\rho c_{p}}$ is the thermal conductivity and $R=\frac{K k^{*}}{4 \sigma T_{\infty}^{3}}$ is the radiation parameter. The physical interest of engineering design quantities is skin friction and Nusselt number, which are defined as

$$
\tau_{w}=\mu\left(\frac{\partial u}{\partial y}\right)_{y=0} \text { and } q_{w}=k\left(\frac{\partial T}{\partial y}\right)_{y=0}
$$

In non-dimensional, skin friction $C_{f}$ and Nusselt number $N u$ can be written as

$$
\begin{aligned}
\frac{C_{f}}{G r^{3 / 4}} & =f^{\prime \prime}(\xi, 0) \\
\frac{N u}{G r^{1 / 4}} & =-\theta^{\prime}(\xi, 0)
\end{aligned}
$$

The location, $\xi \square 0$, corresponds to the vicinity of the lower stagnation point on the cylinder. Since $\frac{\sin \xi}{\xi} \rightarrow \frac{0}{0}$ i.e. 1. For this case the model defined by Equations (15) and (16) reduce to ordinary differential equations.

$$
\begin{aligned}
& f^{\prime \prime \prime}+f f^{\prime \prime}-\left(f^{\prime}\right)^{2}+D e\left[2 f f^{\prime \prime \prime}-\left(f^{\prime \prime}\right)^{2}-f f^{i v}\right]+\theta \frac{\sin (\xi)}{\xi}=0 \\
& \left(1+\frac{4}{3 R}\right) \theta^{\prime \prime}+\operatorname{Pr} f \theta^{\prime}=0
\end{aligned}
$$

\section{Numerical Method of Solution}

The set of differential equations (15) and (16) associated with boundary conditions (17) is highly nonlinear and therefore, it cannot be solved analytically, Hence implicit finite difference method with Keller box scheme has been used for solving it. The main ability of this implicit scheme is to solve systems of differential equations of any order as well as featuring second-order accuracy and attractive extrapolation features. For the implicit finite difference method with Keller box scheme one can refer to Cebeci and Bradshaw (1984) and Bhuvanavijaya et al (2014). We first converted the partial differential equations (15) and (16) into a system of first order differential equations. The resulting equations are written in finite difference forms by considering the functions and their derivatives in terms of center difference. The resulting central finite difference equations are to be linearized by using Newton's method. The obtained linear algebraic equations can be expressed in block diagonal matrix. The solution procedure is given as: 


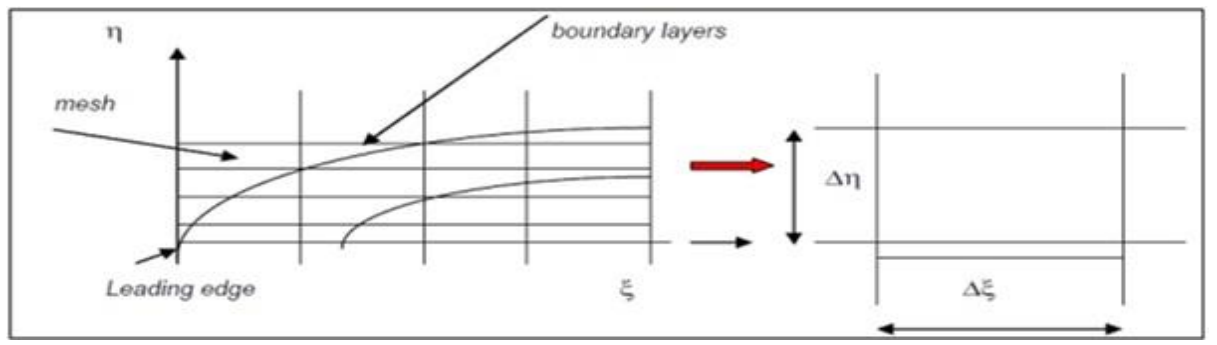

Fig. 2: Grid meshing and a Keller box computation cell

\subsection{Convert the partial differential equations into system of first order equations}

To obtain a set of first order differential equation from (15) and (16) we introduce the new variables $u(\xi, \eta)=f^{\prime}, v(\xi, \eta)=f^{\prime \prime}, w(\xi, \eta)=f^{\prime \prime \prime}, \theta(\xi, \eta)=s, t(\xi, \eta)=s^{\prime}$

$f^{\prime}=u$

$u^{\prime}=v$

$v^{\prime}=w$

$s^{\prime}=t$

$w+f v-u^{2}+D e\left\{2 u w-v^{2}-f w^{\prime}\right\}+s B=\xi\left\{u \frac{\partial f}{\partial \xi}+u \frac{\partial u}{\partial \xi}+D e\left[v \frac{\partial v}{\partial \xi}+w^{\prime} \frac{\partial f}{\partial \xi}-u \frac{\partial w}{\partial \xi}-w \frac{\partial u}{\partial \xi}\right]\right\}$

$\left(1+\frac{4}{3 R}\right) t^{\prime}+\operatorname{Pr} f t=\xi \operatorname{Pr}\left[u \frac{\partial s}{\partial \xi}-t \frac{\partial f}{\partial \xi}\right]$

The corresponding boundary conditions are

$u=0, f=0, s=1$ at $\eta=0$

$u=0, v=0, s=0$ as $\eta \rightarrow \infty$

Where prime denotes differentiation with respect to $\eta$.

\subsection{Write the difference equation using central differences}

As shown in Fig. 2 the mesh points are represented in $\xi, \eta$-plane by $\xi^{n}, \eta_{j}$ where $n=1,2, \ldots . . \mathrm{N}$ and $\mathrm{j}=1,2, \ldots . \mathrm{J}$ and $\eta_{J}=\eta_{\infty}$. The derivates are approximated by centered-difference gradients and averages centered at midpoints of the net, defined by

$$
\begin{aligned}
& \eta_{0}=0, \eta_{j}=\eta_{j-1}+h_{j}, \quad j=1,2,3, \ldots \ldots \ldots \ldots, J, \\
& \eta_{j-1 / 2}=\frac{1}{2}\left(\eta_{j}+\eta_{j-1}\right),(\quad)_{j-1 / 2}=\frac{1}{2}\left(()_{j}+()_{j-1}\right) \\
& \xi^{0}=0, \xi^{n}=\xi^{n-1}+k_{n}, \quad n=1,2,3, \ldots \ldots \ldots, N
\end{aligned}
$$

where $k_{n}, h_{j}$ are respectively $\Delta \xi, \Delta \eta$ spacing. Therefore,

$$
\begin{aligned}
& \frac{f_{j}^{n}-f_{j-1}^{n}}{h_{j}}=\frac{u_{j}^{n}+u_{j-1}^{n}}{2} \\
& \frac{u_{j}^{n}-u_{j-1}^{n}}{h_{j}}=\frac{v_{j}^{n}+v_{j-1}^{n}}{2} \\
& \frac{v_{j}^{n}-v_{j-1}^{n}}{h_{j}}=\frac{w_{j}^{n}+w_{j-1}^{n}}{2}
\end{aligned}
$$




$$
\begin{aligned}
& \frac{s_{j}^{n}-s_{j-1}^{n}}{h_{j}}=\frac{t_{j}^{n}+t_{j-1}^{n}}{2} \\
& v_{j}-v_{j-1}+\frac{h_{j}}{4}(1+\alpha)\left(f_{j}+f_{j-1}\right)\left(v_{j}+v_{j-1}\right)-\frac{h_{j}}{4}(1+\alpha)\left(u_{j}+u_{j-1}\right)^{2}+\frac{h_{j}}{2} D e(1+\alpha)\left(u_{j}+u_{j-1}\right)\left(q_{j}+q_{j-1}\right) \\
& -\frac{h_{j}}{4} D e(1+\alpha)\left(v_{j}+v_{j-1}\right)^{2}-\frac{1}{2} D e(1+\alpha)\left(f_{j}+f_{j-1}\right)\left(q_{j}-q_{j-1}\right)+\frac{h_{j}}{2} \alpha\left(f_{j}+f_{j-1}\right) v_{j-1 / 2}^{n-1} \\
& -\frac{h_{j}}{2} \alpha\left(v_{j}+v_{j-1}\right) f_{j-1 / 2}^{n-1}+\alpha D e\left(q_{j}-q_{j-1}\right) f_{j-1 / 2}^{n-1}-\frac{h_{j}}{2}\left(f_{j}+f_{j-1}\right)\left(q^{\prime}\right)_{j-1 / 2}^{n-1}=\left(R_{1}\right)_{j-1 / 2}^{n-1} \\
& \quad\left(1+\frac{4}{3 R}\right)\left(t_{j}-t_{j-1}\right)+\frac{h_{j}}{4}(1+\alpha)\left(f_{j}+f_{j-1}\right)\left(t_{j}+t_{j-1}\right)-\frac{h_{j}}{4} \alpha\left(u_{j}+u_{j-1}\right)\left(s_{j}+s_{j-1}\right) \\
& \quad+\frac{h_{j}}{2} \alpha\left(s_{j}+s_{j-1}\right) u_{j-1 / 2}^{n-1}-\frac{h_{j}}{2} \alpha\left(u_{j}+u_{j-1}\right) s_{j-1 / 2}^{n-1}+\frac{h_{j}}{2} \alpha\left(t_{j}+t_{j-1}\right) f_{j-1 / 2}^{n-1} \\
& \quad-\frac{h_{j}}{2}\left(f_{j}+f_{j-1}\right) t_{j-1 / 2}^{n-1}=\left(R_{2}\right)_{j-1 / 2}^{n-1}
\end{aligned}
$$

where

$$
\begin{aligned}
& \left(R_{1}\right)_{j-1 / 2}^{n-1}=-h_{j}\left[\begin{array}{l}
v_{j-1 / 2}^{\prime}+(1-\alpha)(f v)_{j-1 / 2}-(1-\alpha)\left(u_{j-1 / 2}\right)^{2}+2 D e(1-\alpha)(u q)_{j-1 / 2}-D e(1-\alpha)\left(v_{j-1 / 2}\right)^{2} \\
-D e(1-\alpha)\left(f q^{\prime}\right)_{j-1 / 2}+B s_{j-1 / 2}^{n-1}
\end{array}\right]^{-1} \\
& \left(R_{2}\right)_{j-1 / 2}^{n-1}=-h_{j}\left[\begin{array}{l}
\left(1+\frac{4}{3 R}\right)\left(\frac{t_{j}-t_{j-1}}{h_{j}}\right)+(f t)_{j-1 / 2} \\
+\alpha(u s)_{j-1 / 2}-\alpha(f t)_{j-1 / 2}
\end{array}\right]^{n-1}
\end{aligned}
$$

The boundary conditions are

$$
f_{0}^{n}=0, u_{0}^{n}=0, s_{0}^{n}=1, u_{J}^{n}=0, v_{J}^{n}=0, s_{J}^{n}=0
$$

\subsection{Linearize the resulting algebraic equations by Newton's method}

To linearize the system of nonlinear equations (23) using Newton's method, we introduce the following iterates $f_{j}^{i+1}=f_{j}^{i}+\delta f_{j}^{i}$,

$u_{j}^{i+1}=u_{j}^{i}+\delta u_{j}^{i}$,

$v_{j}^{i+1}=v_{j}^{i}+\delta v_{j}^{i}$,

$w_{j}^{i+1}=w_{j}^{i}+\delta w_{j}^{i}$

$u_{j}^{i+1}=s_{j}^{i}+\delta s_{j}^{i}$,

$t_{j}^{i+1}=t_{j}^{i}+\delta t_{j}^{i}$

By using Eq. (24), Eq. (23) becomes

$$
\begin{aligned}
& \delta f_{j}-\delta f_{j-1}-\frac{1}{2} h_{j}\left(\delta u_{j}+\delta u_{j-1}\right)=\left(r_{1}\right)_{j-1 / 2} \\
& \delta u_{j}-\delta u_{j-1}-\frac{1}{2} h_{j}\left(\delta v_{j}+\delta v_{j-1}\right)=\left(r_{2}\right)_{j-1 / 2} \\
& \delta v_{j}-\delta v_{j-1}-\frac{1}{2} h_{j}\left(\delta w_{j}+\delta w_{j-1}\right)=\left(r_{3}\right)_{j-1 / 2} \\
& \delta s_{j}-\delta s_{j-1}-\frac{1}{2} h_{j}\left(\delta t_{j}+\delta t_{j-1}\right)=\left(r_{4}\right)_{j-1 / 2}
\end{aligned}
$$




$$
\begin{aligned}
& \left(a_{1}\right)_{j} \delta w_{j}+\left(a_{2}\right)_{j} \delta w_{j-1}+\left(a_{3}\right)_{j} \delta f_{j}+\left(a_{4}\right)_{j} \delta f_{j-1}+\left(a_{5}\right)_{j} \delta u_{j}+\left(a_{6}\right)_{j} \delta u_{j-1} \\
& +\left(a_{7}\right)_{j} \delta v_{j}+\left(a_{8}\right)_{j} \delta v_{j-1}+\left(a_{9}\right)_{j} \delta s_{j}+\left(a_{10}\right)_{j} \delta s_{j-1}=\left(r_{5}\right)_{j-1 / 2} \\
& \left(b_{1}\right)_{j} \delta t_{j}+\left(b_{2}\right)_{j} \delta t_{j-1}+\left(b_{3}\right)_{j} \delta f_{j}+\left(b_{4}\right)_{j} \delta f_{j-1}+\left(b_{5}\right)_{j} \delta u_{j}+\left(b_{6}\right)_{j} \delta u_{j-1} \\
& +\left(b_{7}\right)_{j} \delta s_{j}+\left(b_{8}\right)_{j} \delta s_{j-1}=\left(r_{6}\right)_{j-1 / 2}
\end{aligned}
$$

Where

$$
\begin{aligned}
& \left(a_{1}\right)_{j}=\frac{h_{j}}{2}+D e(1+\alpha)\left(h_{j} u_{j-1 / 2}-2 f_{j-1 / 2}\right)+\frac{\alpha D e}{2} f_{j-1 / 2}^{n-1}, \\
& \left(a_{2}\right)_{j}=\frac{h_{j}}{2}+D e(1+\alpha)\left(h_{j} u_{j-1 / 2}+2 f_{j-1 / 2}\right)+\frac{\alpha D e}{2} f_{j-1 / 2}^{n-1} \\
& \left(a_{3}\right)_{j}=\frac{h_{j}}{2} v_{j-1 / 2}-\frac{D e}{2}(1+\alpha)\left(w_{j}-w_{j-1}\right)-\frac{\alpha h_{j}}{2}\left(u_{j-1 / 2}^{n-1}+u_{j-1 / 2}+D e\left(\frac{w_{j}^{n-1}-w_{j-1}^{n-1}}{h_{j}}\right)\right) \\
& \left(a_{5}\right)_{j}=(1+\alpha) \frac{h_{j}}{2} u_{j-1 / 2}+D e(1+\alpha) h_{j} w_{j-1 / 2}+\alpha \frac{h_{j}}{2}\left(f_{j-1 / 2}^{n-1}+f_{j-1 / 2}\right),\left(a_{4}\right)_{j}=\left(a_{3}\right)_{j},\left(a_{6}\right)_{j}=\left(a_{5}\right)_{j} \text {, } \\
& \left(a_{7}\right)_{j}=h_{j}\left[\frac{1}{2} f_{j-1 / 2}-\operatorname{De}(1+\alpha) v_{j-1 / 2}\right],\left(a_{8}\right)_{j}=\left(a_{7}\right)_{j},\left(a_{9}\right)_{j}=\left(a_{10}\right)_{j}=\frac{B h_{j}}{2} \\
& \left(b_{1}\right)_{j}=\left(1+\frac{4}{3 R}\right)+h_{j}\left[\frac{\operatorname{Pr}}{2}(1+\alpha) f_{j-1 / 2}+\alpha \operatorname{Pr} f_{j-1 / 2}^{n-1}\right] \text {, } \\
& \left(b_{2}\right)_{j}=-\left(1+\frac{4}{3 R}\right)+h_{j}\left[\frac{\operatorname{Pr}}{2}(1+\alpha) f_{j-1 / 2}+\alpha \operatorname{Pr} f_{j-1 / 2}^{n-1}\right],\left(b_{3}\right)_{j}=\left(b_{4}\right)_{j}=h_{j} \operatorname{Pr}\left[\frac{1+\alpha}{2} t_{j-1 / 2}-t_{j-1 / 2}^{n-1}\right] \\
& \left(b_{5}\right)_{j}=\left(b_{6}\right)_{j}=-h_{j} \operatorname{Pr} \alpha\left[\frac{s_{j-1 / 2}}{2}+s_{j-1 / 2}^{n-1}\right], \quad\left(b_{7}\right)_{j}=\left(b_{8}\right)_{j}=-\frac{h_{j} \operatorname{Pr} \alpha}{2}\left[u_{j-1 / 2}+u_{j-1 / 2}^{n-1}\right] \\
& \left(r_{1}\right)_{j-1 / 2}=f_{j-1}-f_{j}+h_{j} u_{j-1 / 2},\left(r_{2}\right)_{j-1 / 2}=u_{j-1}-u_{j}+h_{j} v_{j-1 / 2}, \\
& \left(r_{3}\right)_{j-1 / 2}=v_{j-1}-v_{j}+h_{j} w_{j-1 / 2},\left(r_{4}\right)_{j-1 / 2}=s_{j-1}-s_{j}+h_{j} t_{j-1 / 2} \\
& \left(r_{5}\right)_{j-1 / 2}=\left(R_{1}\right)_{j-1 / 2}-h_{j}\left(w_{j-1 / 2}+f_{j-1 / 2} v_{j-1 / 2}-(1+\alpha)\left(u_{j-1 / 2}\right)^{2}\right) \\
& -(1+\alpha) u_{j-1 / 2} w_{j-1 / 2}-h_{j} 2 D e\left(\left(v_{j-1 / 2}\right)^{2}+f_{j-1 / 2}\left(\frac{w_{j}-w_{j-1}}{h_{j}}\right)\right) \\
& -h_{j} \alpha\left(f_{j-1 / 2}^{n-1} u_{j-1 / 2}-u_{j-1 / 2}^{n-1} f_{j-1 / 2}+f_{j-1 / 2} u_{j-1 / 2}\right) \\
& -\alpha h_{j} D e\left(\frac{w_{j}^{n-1}-w_{j-1}^{n-1}}{h_{j}} f_{j-1 / 2}+f_{j-1 / 2}^{n-1} \frac{w_{j}-w_{j-1}}{h_{j}}\right)+B s_{j-1 / 2} \\
& \left(r_{6}\right)_{j-1 / 2}=\left(R_{2}\right)_{j-1 / 2}-\left(1+\frac{4}{3 R}\right)\left(t_{j}-t_{j-1}\right)-h_{j}\left[\begin{array}{l}
\operatorname{Pr}(1+\alpha) f_{j-1 / 2} t_{j-1 / 2} \\
\left.-\alpha \operatorname{Pr}\left(\begin{array}{l}
u_{j-1 / 2} s_{j-1 / 2}+u_{j-1 / 2} s_{j-1 / 2}^{n-1}-u_{j-1 / 2}^{n-1} s_{j-1 / 2} \\
+t_{j-1 / 2}^{n-1} f_{j-1 / 2}-f_{j-1 / 2}^{n-1} t_{j-1 / 2}
\end{array}\right)\right]
\end{array}\right.
\end{aligned}
$$
where $\alpha=\frac{\xi^{n-1 / 2}}{k_{n}}, \quad B=\frac{\operatorname{Sin}\left(\xi^{n-1 / 2}\right)}{\xi^{n-1 / 2}}$

To complete the system (25) we recall the boundary conditions (24g), which can be satisfied exactly with no iteration. So, to maintain these correct values in all the iterates, we take 


$$
\begin{aligned}
& \delta u_{0}=0, \quad \delta f_{0}=0, \quad \delta s_{0}=0 \\
& \delta u_{J}=0, \delta v_{J}=0, \delta s_{J}=0 .
\end{aligned}
$$

\subsection{Block elimination method}

The linear system (25) can now be solved by the block-elimination method, as it is explained in $\mathrm{Na}$ (1979). The linearized difference equations of the system (25) have a block tri-diagonal structure. In a matrix vector form, this can be written as

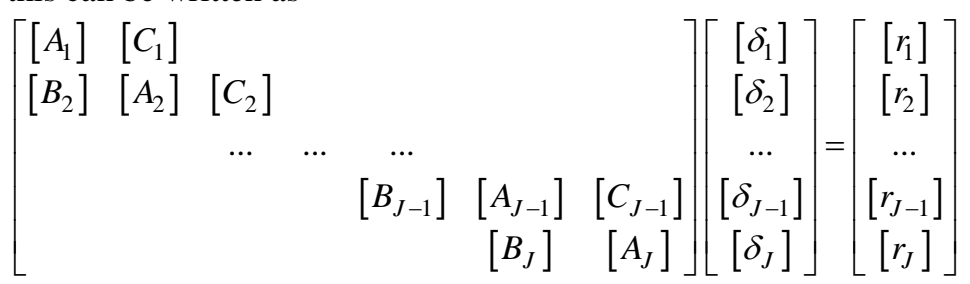

That is: $A \delta=r$

where the elements are defined by

$$
\begin{aligned}
& {\left[A_{1}\right]=\left[\begin{array}{cccccc}
0 & 0 & 0 & 1 & 0 & 0 \\
d_{1} & 0 & 0 & 0 & 0 & 0 \\
-1 & d_{1} & 0 & 0 & d_{1} & 0 \\
0 & 0 & d_{1} & 0 & 0 & d_{1} \\
\left(a_{8}\right)_{1} & \left(a_{2}\right)_{1} & 0 & \left(a_{3}\right)_{1} & \left(a_{1}\right)_{1} & 0 \\
0 & 0 & \left(b_{2}\right)_{1} & \left(b_{3}\right)_{1} & 0 & \left(b_{1}\right)_{1}
\end{array}\right], d_{1}=\frac{h_{1}}{2}} \\
& {\left[A_{j}\right]=\left[\begin{array}{cccccc}
d_{j} & 0 & 0 & 1 & 0 & 0 \\
-1 & d_{j} & 0 & 0 & 0 & 0 \\
0 & -1 & 0 & 0 & d_{j} & 0 \\
0 & 0 & -1 & 0 & 0 & d_{j} \\
\left(a_{6}\right)_{j} & \left(a_{8}\right)_{j} & \left(a_{10}\right)_{j} & \left(a_{3}\right)_{j} & \left(a_{1}\right)_{j} & 0 \\
\left(b_{6}\right)_{j} & 0 & \left(b_{8}\right)_{j} & \left(b_{3}\right)_{j} & 0 & \left(b_{1}\right)_{j}
\end{array}\right], d_{j}=\frac{h_{j}}{2}, 2 \leq j \leq J} \\
& {\left[B_{j}\right]=} \\
& {\left[C_{j}\right]=\left[\begin{array}{ccccccc}
0 & 0 & 0 & -1 & 0 & 0 \\
0 & 0 & 0 & 0 & 0 & 0 \\
0 & 0 & 0 & 0 & d_{j} & 0 \\
0 & 0 & 0 & 0 & 0 & d_{j} \\
0 & 0 & 0 & \left(a_{4}\right)_{j} & \left(a_{2}\right)_{j} & 0 \\
0 & 0 & 0 & \left(b_{4}\right)_{j} & 0 & \left(b_{2}\right)_{j}
\end{array}\right], d_{j}=\frac{h_{j}}{2}, 2 \leq j \leq J} \\
& 0 \\
& 0
\end{aligned}
$$




$$
\left[\delta_{1}\right]=\left[\begin{array}{l}
\delta u_{0} \\
\delta v_{0} \\
\delta s_{0} \\
\delta f_{1} \\
\delta w_{1} \\
\delta t_{1}
\end{array}\right],\left[\delta_{j}\right]=\left[\begin{array}{l}
\delta u_{j-1} \\
\delta v_{j-1} \\
\delta s_{j-1} \\
\delta f_{j} \\
\delta w_{j} \\
\delta t_{j}
\end{array}\right], 2 \leq j \leq J,\left[r_{j}\right]=\left[\begin{array}{c}
\left(r_{1}\right)_{j-1.2} \\
\left(r_{2}\right)_{j-1.2} \\
\left(r_{3}\right)_{j-1.2} \\
\left(r_{4}\right)_{j-1.2} \\
\left(r_{5}\right)_{j-1.2} \\
\left(r_{6}\right)_{j-1.2}
\end{array}\right], 1 \leq j \leq J,
$$

The coefficient matrix A in (26) has a block tri-diagonal structure and the difference equations are solved using a block matrix version of the Thomas algorithm. The numerical results are strongly influenced by the number of mesh points in $\xi, \eta$-directions. After some trials in the $\eta$-direction a larger number of mesh points are selected whereas in the $\xi$-direction significantly less mesh points are necessary. $\eta_{\max }$ has been set at appropriate position and this constitutes an adequately large value at which the prescribed boundary conditions are satisfied. $\xi_{\max }$ is set at 2.5 for the simulations. Mesh independence has been comfortably attained in the simulations. The numerical algorithm is executed in MATLAB®.

\subsection{Grid independency test}

In order to check the effects of step size $(\Delta \eta)$ we found the Nusselt number and Sherwood number with four different step sizes as $\Delta \eta=0.1, \Delta \eta=0.01$ and $\Delta \eta=0.001$. We observe from Table 1 that the results are independent with the step size $(\Delta \eta)$ and exist with increasing CPU time (sec). Hence a step size $\Delta \eta=0.01$ is selected to be satisfactory for a convergence criterion of $10^{-5}$ in all cases.

\subsection{Code validation}

In order to assess the present numerical method we compare present results with previously published work by Merkin (1977) and Yih (2000) and good agreement is obtained as shown in Table 2.

Table 1: Grid-independence for $\operatorname{Pr}=10, \mathrm{De}=1$ and $\mathrm{R}=1$

\begin{tabular}{|c|c|c|c|}
\hline$\Delta \eta$ (Step size) & $-f^{\prime \prime}(\xi, 0)$ & $-\theta^{\prime}(\xi, 0)$ & CPU time (Sec) \\
\hline 0.1 & 0.6711096013 & 0.52420031235 & 1.0140 \\
\hline 0.01 & 0.67110538432 & 0.52420013563 & 10.4833 \\
\hline 0.001 & 0.6711032946 & 0.52420009563 & 25.8026 \\
\hline
\end{tabular}

Table 2: Values of Nusselt number for different values of stream wise coordinate with $\mathrm{De}=0, \operatorname{Pr}=10$ and $\mathrm{R}=0$

\begin{tabular}{|l|l|l|l|}
\hline$\xi$ & $\begin{array}{l}\text { Merkin } \\
(1977)\end{array}$ & $\begin{array}{l}\text { Yih } \\
(2000)\end{array}$ & $\begin{array}{l}\text { Present } \\
\text { Results }\end{array}$ \\
\hline 0.0 & 0.4212 & 0.4214 & 0.4213 \\
\hline 1.0 & 0.4025 & 0.4030 & 0.4031 \\
\hline 2.0 & 0.3443 & 0.3457 & 0.3458 \\
\hline 3.0 & 0.2252 & 0.2267 & 0.2261 \\
\hline
\end{tabular}

\section{Results and Discussion}

Comprehensive solutions have been obtained and are presented in Figs. 3-14 and in Tables 2-4. Numerical calculations have been carried out for different values of Deborah number (De), Prandtl number (Pr) and 
Radiation parameter $(\mathrm{R})$ on flow velocity and temperature profiles, skin friction and Nusselt number. Throughout the calculation we have fixed the values of $\mathrm{De}=1, \operatorname{Pr}=10$ and $\mathrm{R}=1$.

\subsection{Variation of Deborah number}

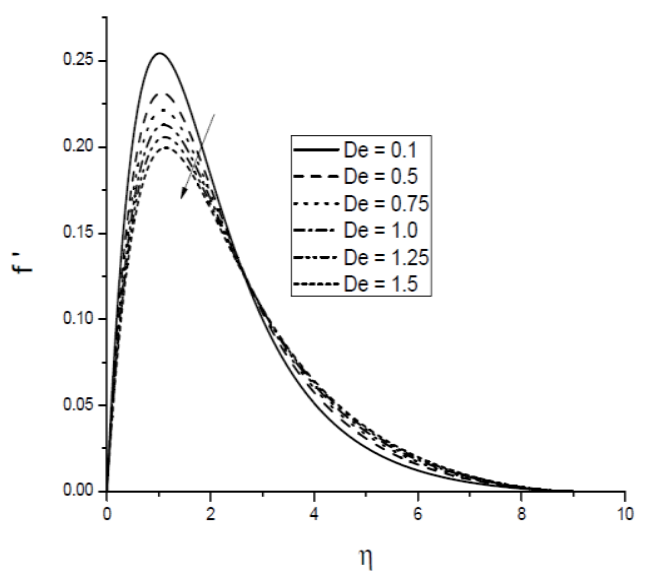

Fig. 3: Velocity profile for different values of De

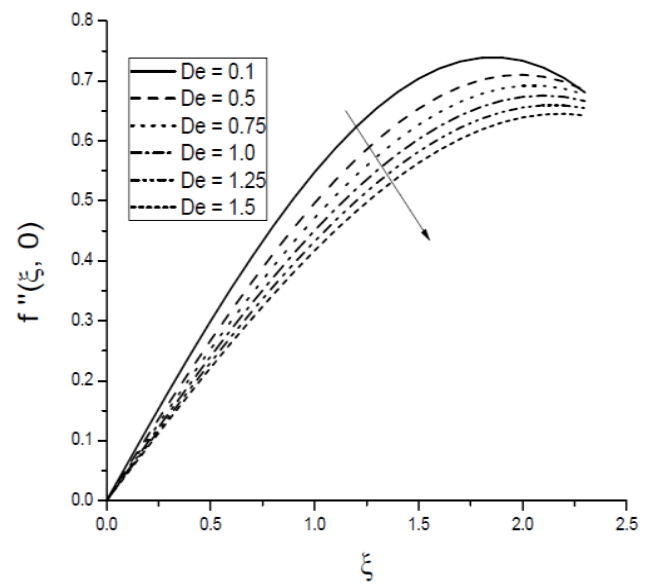

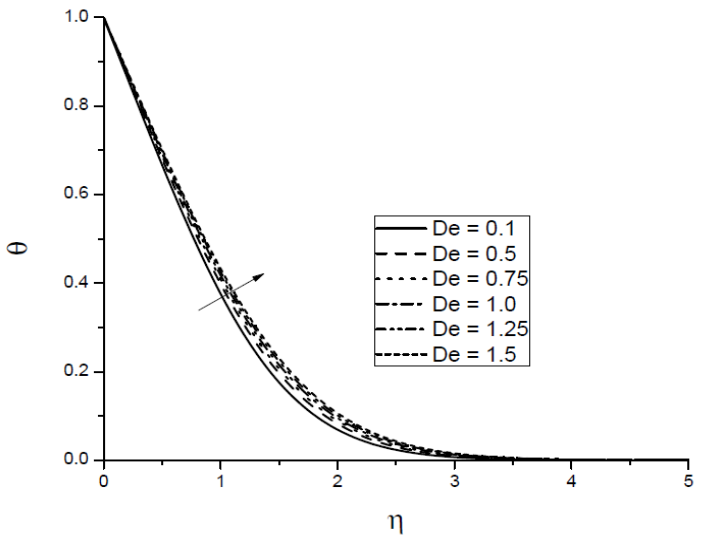

Fig. 4: Temperature profile for different values of De

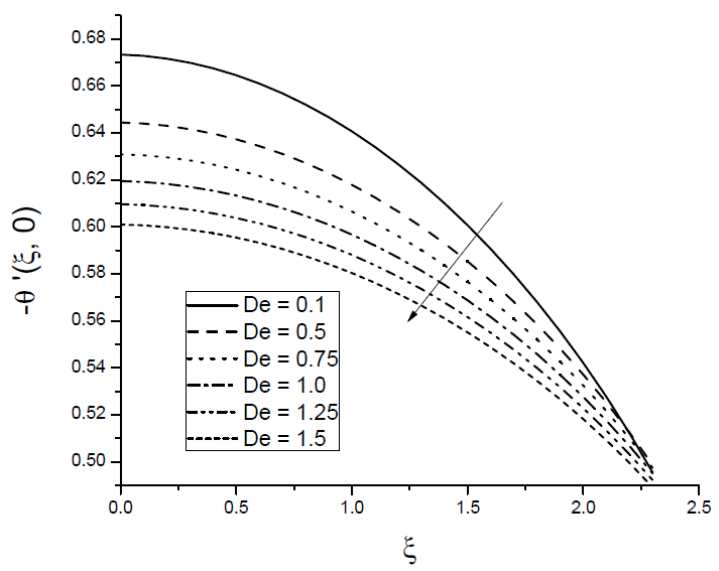

Fig. 5: Skin friction coefficient for different values of De. Fig. 6: Nusselt number for different values of De

Table 3: Values of $f^{\prime \prime}(\xi, 0)$ and $-\theta^{\prime}(\xi, 0)$ for different values of $\operatorname{De}$ for $\operatorname{Pr}=10$ and $\mathrm{R}=1.0$

\begin{tabular}{|c|c|c|c|c|c|c|c|c|}
\hline & \multicolumn{2}{|c|}{$\xi=0$} & \multicolumn{2}{c|}{$\xi=0.5$} & \multicolumn{2}{c|}{$\xi=1$} & \multicolumn{2}{c|}{$\xi=1.5$} \\
\hline De & $f^{\prime \prime}(\xi, 0)$ & $-\theta^{\prime}(\xi, 0)$ & $f^{\prime \prime}(\xi, 0)$ & $-\theta^{\prime}(\xi, 0)$ & $f^{\prime \prime}(\xi, 0)$ & $-\theta^{\prime}(\xi, 0)$ & $f^{\prime \prime}(\xi, 0)$ & $-\theta^{\prime}(\xi, 0)$ \\
\hline 0.1 & 0 & 0.6734 & 0.2992 & 0.6645 & 0.5479 & 0.6407 & 0.7039 & 0.6007 \\
\hline 0.5 & 0 & 0.6444 & 0.2672 & 0.6373 & 0.4963 & 0.6179 & 0.6539 & 0.5853 \\
\hline 0.75 & 0 & 0.6309 & 0.2526 & 0.6243 & 0.4715 & 0.6065 & 0.6269 & 0.5766 \\
\hline 1.0 & 0 & 0.6195 & 0.2405 & 0.6133 & 0.4506 & 0.5967 & 0.6302 & 0.5687 \\
\hline 1.5 & 0 & 0.6010 & 0.2215 & 0.5954 & 0.4170 & 0.5803 & 0.5633 & 0.5551 \\
\hline 2.0 & 0 & 0.5863 & 0.2069 & 0.5811 & 0.3908 & 0.5671 & 0.5312 & 0.5436 \\
\hline
\end{tabular}

Figs. $3 \& 4$ represent velocity and temperature profiles for different values of Deborah number. We observe that increase in De tends to decrease maximum velocity profile near the surface, while it increases far away from the cylinder surface. It is because of increase in De means increase in elasticity and decrease in viscosity of the fluid reduces the velocity boundary layer thickness. Increase in Deborah number results an enhancement in 
temperature profile as shown in Fig. 4. Further it is clear that thermal boundary layer thickness increases with increase in De. For different values of Deborah number De, the skin friction coefficient and Nusselt number (rate of heat transfer) are shown in Figs. $5 \& 6$ and Table 3. Both skin friction coefficient and Nusselt number are decreased with increase in Deborah number. As explained above, increase in De results depreciation in velocity gradient. The numerical values of average Nusselt number for various values of Deborah number are shown in Table 4. This shows that increase in Deborah number leads to depreciation in the average Nusselt number.

Table 4: Average Nusselt number for different values of De for $\mathrm{R}=2$ and $\operatorname{Pr}=10$

\begin{tabular}{|l|l|l|l|l|l|}
\hline De & $\xi=0.1$ & $\xi=0.5$ & $\xi=1.0$ & $\xi=1.5$ & $\xi=2.0$ \\
\hline 0.1 & 0.0560 & 0.2792 & 0.6627 & 1.0026 & 1.3182 \\
\hline 0.5 & 0.0538 & 0.2681 & 0.6373 & 0.9669 & 1.2763 \\
\hline 0.75 & 0.0527 & 0.2628 & 0.6250 & 0.9492 & 1.2549 \\
\hline 1.0 & 0.0518 & 0.2582 & 0.6145 & 0.9339 & 1.2360 \\
\hline
\end{tabular}

\subsection{Variation of Prandtl number}

Figs. $7 \& 8$ illustrate velocity and temperature profile along the radial direction, which is normal to the circular cylinder, for different values of Prandtl number. From these figures it is observed that velocity and temperature profiles are reduced with increase in Pr, and therefore it turns to reduce velocity and thermal boundary layer thickness. The reason is that for higher values of Pr, heat is able to diffuse far away from the heated surface more rapidly and thermal boundary layer is thicker for smaller values of Pr. The numerical results of skin friction coefficient and Nusselt number are displayed graphically in Figs. $9 \& 10$ and table 5 for different values of Pr. It is noticed from the figures that increase in Prandtl number accelerates, i.e. increase in skin friction coefficient and decelerates the Nusselt number. The results of average Nusselt number for different values of Prandtl number Pr presented in Table 6. From these values we observed that there is an enhancement in average Nusselt number with increase in Pr.

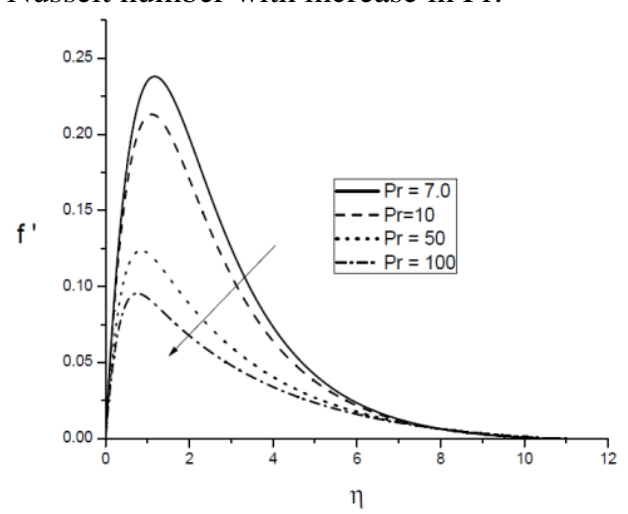

Fig. 7: Velocity profile for different values of $\mathrm{Pr}$

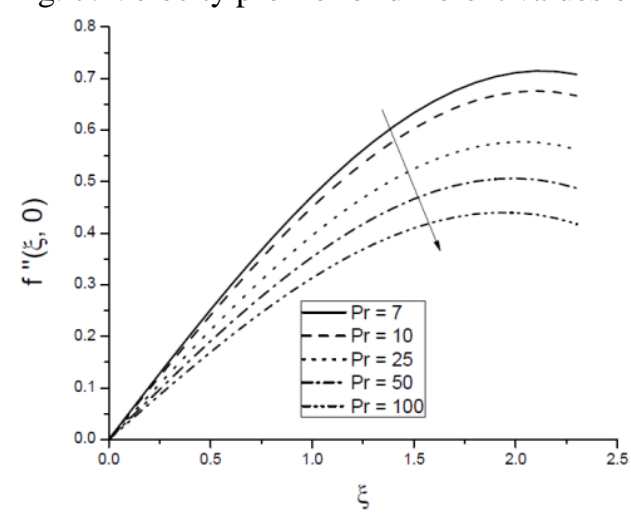

Fig. 9: Skin friction coefficient for different values of $\mathrm{Pr}$

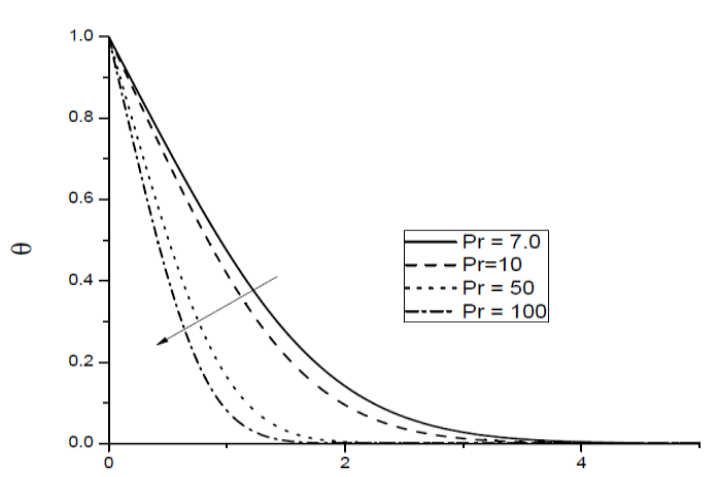

$\eta$

Fig. 8: Temperature profile for different values of $\mathrm{Pr}$

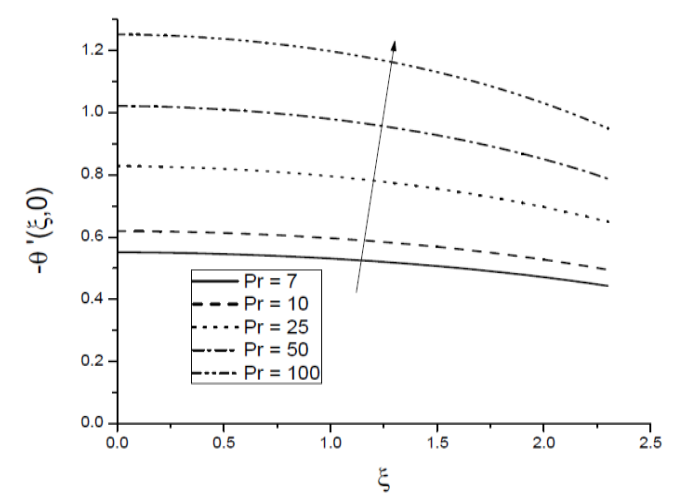

Fig. 10: Nusselt number for different values of $\mathrm{Pr}$ 
Table 5: Values of $f^{\prime \prime}(\xi, 0)$ and $-\theta^{\prime}(\xi, 0)$ for different values of $\operatorname{Pr}$ for $\mathrm{De}=1.0$ and $\mathrm{R}=1.0$

\begin{tabular}{|c|c|c|c|c|c|c|c|c|}
\hline & \multicolumn{2}{|c|}{$\xi=0$} & \multicolumn{2}{c|}{$\xi=0.5$} & \multicolumn{2}{c|}{$\xi=1$} & \multicolumn{2}{c|}{$\xi=1.5$} \\
\hline $\operatorname{Pr}$ & $f^{\prime \prime}(\xi, 0)$ & $-\theta^{\prime}(\xi, 0)$ & $f^{\prime \prime}(\xi, 0)$ & $-\theta^{\prime}(\xi, 0)$ & $f^{\prime \prime}(\xi, 0)$ & $-\theta^{\prime}(\xi, 0)$ & $f^{\prime \prime}(\xi, 0)$ & $-\theta^{\prime}(\xi, 0)$ \\
\hline 0.01 & 0 & 0.0945 & 0.3447 & 0.0943 & 0.6550 & 0.0940 & 0.9005 & 0.0934 \\
\hline 0.71 & 0 & 0.2428 & 0.3111 & 0.2406 & 0.5889 & 0.2346 & 0.8040 & 0.2247 \\
\hline 7.0 & 0 & 0.5509 & 0.2513 & 0.5456 & 0.4716 & 0.5311 & 0.6333 & 0.5068 \\
\hline 10 & 0 & 0.6195 & 0.2405 & 0.6133 & 0.4506 & 0.5967 & 0.6032 & 0.5687 \\
\hline 50 & 0 & 1.0218 & 0.1905 & 1.0105 & 0.3539 & 0.9798 & 0.4663 & 0.9278 \\
\hline 100 & 0 & 1.2522 & 0.1692 & 1.2376 & 0.3132 & 1.1980 & 0.4099 & 1.1309 \\
\hline
\end{tabular}

Table 6: Average Nusselt number for different values of $\operatorname{Pr}$ for $\mathrm{De}=1$ and $\mathrm{R}=1$

\begin{tabular}{|l|l|l|l|l|l|}
\hline $\operatorname{Pr}$ & $\xi=0.1$ & $\xi=0.5$ & $\xi=1.0$ & $\xi=1.5$ & $\xi=2.0$ \\
\hline 0.71 & 0.0182 & 0.0908 & 0.2164 & 0.3293 & 0.4369 \\
\hline 7 & 0.0413 & 0.2061 & 0.4907 & 0.7461 & 0.9885 \\
\hline 10 & 0.0465 & 0.2317 & 0.5516 & 0.8385 & 1.1103 \\
\hline 50 & 0.0766 & 0.3821 & 0.9086 & 1.3789 & 1.8210 \\
\hline
\end{tabular}

\subsection{Variation of Radiation Parameter}

Figs. $11 \& 12$ show the behavior of velocity and temperature for different values of Radiation parameter. It is noticed that increase in radiation $(\mathrm{R})$ tends to depreciation in velocity and temperature profiles within the boundary layer, as well as reduced velocity and thermal boundary layer thickness. Figs. 13 and 14 and Table 7 shows the results of skin friction coefficient and Nusselt number for different values of radiation parameter R. It is clear that the skin friction coefficient decreases, while Nusselt number increases with increasing values of radiation parameter $\mathrm{R}$.

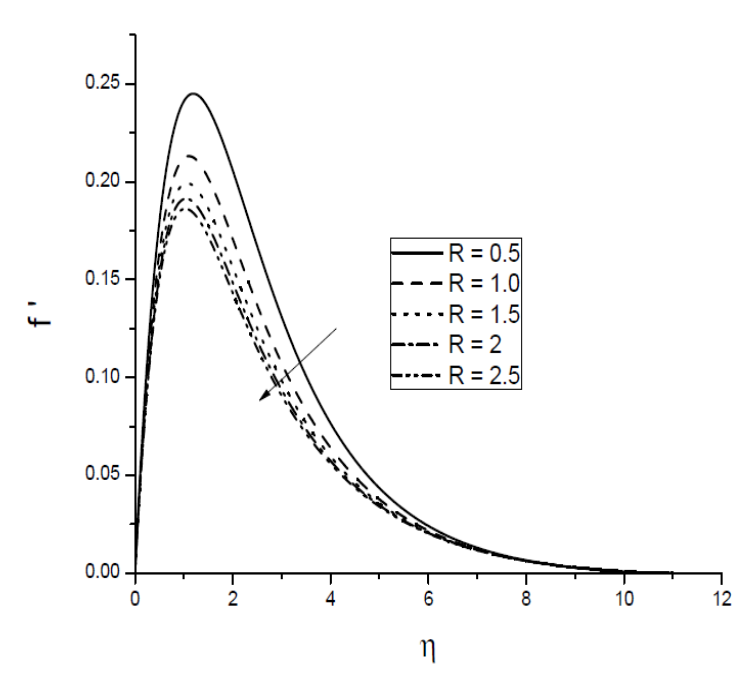

Fig. 11: Velocity profile for different values of $\mathrm{R}$

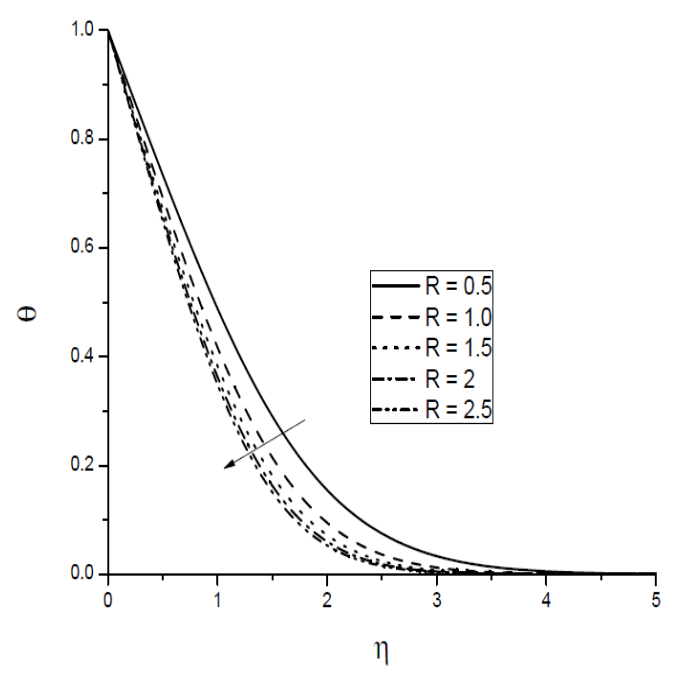

Fig. 12: Temperature profile for different values of $\mathrm{R}$

\subsection{Variation of Stream Wise Coordinate $(\xi)$}

Figures 15 and 16 represent variation of transverse (stream wise) coordinate $(\xi)$, on velocity and temperature distributions respectively. Generally velocity is noticeably lowered with increasing migration from the leading edge i.e. larger $\xi$ values (Fig. 15). The maximum velocity is computed at the lower stagnation point $(\xi \sim 0)$ for low values of radial coordinate $(\eta)$. The transverse coordinate clearly exerts a significant influence on momentum development. A very strong increase in temperature $(\theta)$, as observed in figure 16, is generated 
throughout the boundary layer with increasing $\xi$ values. The temperature field decays monotonically. Temperature is maximized at the surface of the cylindrical body $(\eta=0$, for all $\xi)$ and minimized in the free stream $(\eta=5)$. Although the behavior at the upper stagnation point $(\xi \sim 2.0)$ is not computed, the pattern in figure $8 \mathrm{~b}$ suggests that temperature will continue to progressively grow here compared with previous locations on the cylinder surface (lower values of $\xi$ ). It is an important to note that all the numerical results presented here should be claimed and are accurate and acceptable. These methods can easily be extended to more complicated heat transfer problems.

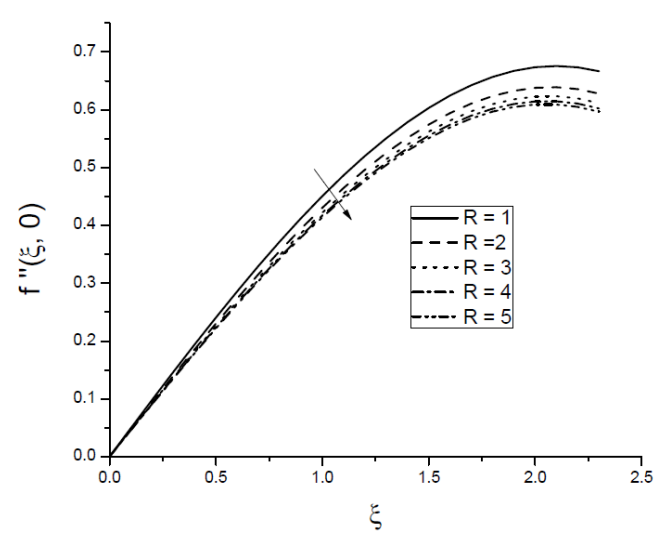

Fig. 13: Skin friction coefficient for different values of $\mathrm{R}$

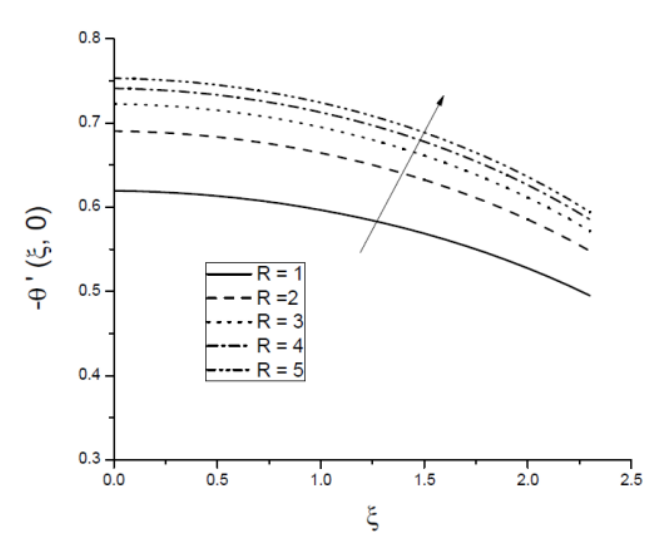

Fig. 14: Nusselt number for different values of $\mathrm{R}$

Table 7: Values of $f^{\prime \prime}(\xi, 0)$ and $-\theta^{\prime}(\xi, 0)$ for different values of $\mathrm{R}$ for $\mathrm{De}=1.0$ and $\mathrm{Pr}=10$

\begin{tabular}{|c|c|c|c|c|c|c|c|c|}
\hline & \multicolumn{2}{|c|}{$\xi=0$} & \multicolumn{2}{c|}{$\xi=0.5$} & \multicolumn{2}{c|}{$\xi=1$} & \multicolumn{2}{c|}{$\xi=1.5$} \\
\hline $\mathrm{R}$ & $f^{\prime \prime}(\xi, 0)$ & $-\theta^{\prime}(\xi, 0)$ & $f^{\prime \prime}(\xi, 0)$ & $-\theta^{\prime}(\xi, 0)$ & $f^{\prime \prime}(\xi, 0)$ & $-\theta^{\prime}(\xi, 0)$ & $f^{\prime \prime}(\xi, 0)$ & $-\theta^{\prime}(\xi, 0)$ \\
\hline 0.5 & 0 & 0.5337 & 0.2541 & 0.5286 & 0.4771 & 0.5146 & 0.6412 & 0.4912 \\
\hline 1.0 & 0 & 0.6195 & 0.2405 & 0.6133 & 0.4506 & 0.5967 & 0.6032 & 0.5687 \\
\hline 1.5 & 0 & 0.6633 & 0.2341 & 0.6566 & 0.4380 & 0.6385 & 0.5852 & 0.6081 \\
\hline 2.0 & 0 & 0.6904 & 0.2302 & 0.6834 & 0.4305 & 0.6644 & 0.5745 & 0.6325 \\
\hline 2.5 & 0 & 0.7089 & 0.2276 & 0.7017 & 0.4255 & 0.6821 & 0.5673 & 0.6491 \\
\hline
\end{tabular}

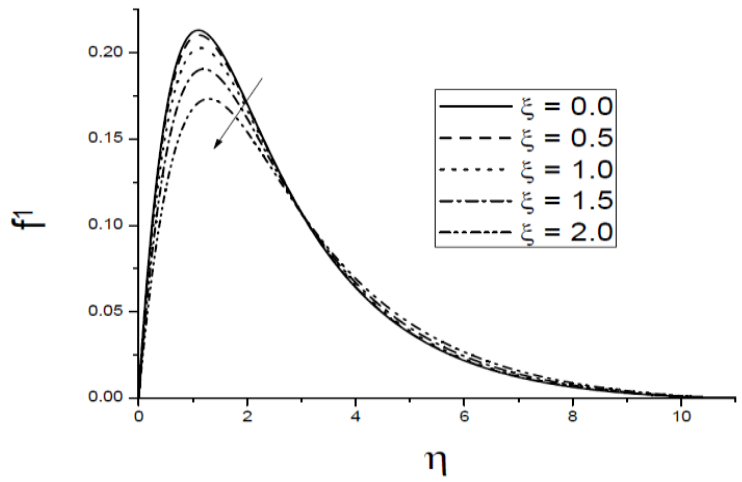

Fig. 15: Velocity profile for different values of $\xi$

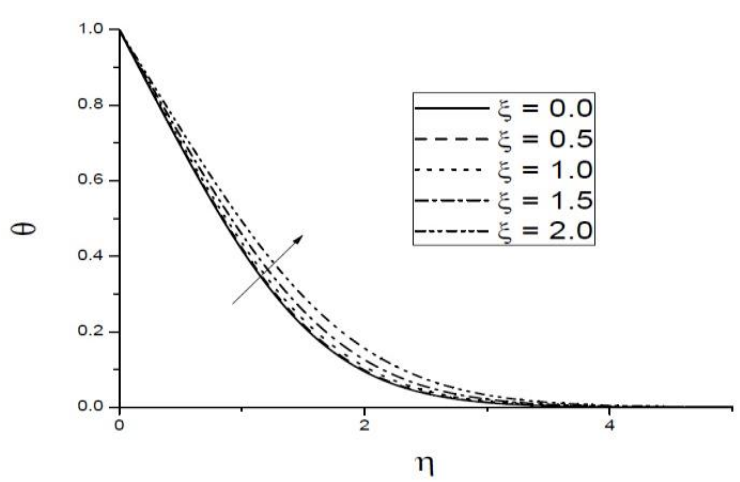

Fig. 16: Temperature profile for different values of $\xi$ 


\section{Conclusion}

A mathematical model has been investigated for free convection boundary layer flow of non-Newtonian second grade fluid past an impermeable isothermal horizontal cylinder. The governing boundary layer equations for mass, momentum and energy are transformed into non-dimensional equations by using the non-similarity transformations and then found the numerical solutions by employing an efficient, validated implicit finite difference method. The results of various physical parameters have shown that:

- Increasing the Deborah number reduces velocity profile, skin friction and Nusselt number where as it enhances the temperature profile.

- Increasing Prandtl number decelerates the flow velocity, temperature and skin friction but Nusselt number enhances considerably.

- Increase in radiation parameter retards the flow velocity, temperature and skin friction. But Nusselt number enhances markedly with increase in radiation parameter.

\section{Acknowledgements}

One of the authors Dr. B. Mallikarjuna wishes to thank to B.M.S. College of Engineering, Bangalore-19, for giving support to made this paper through the Technical Education Quality Improvement Programme [TEQIPII] of the MHRD, Government of India.

\section{Reference}

Beard, D.W. and Walter, K. (1964): Elastico - viscous boundary layer flows. Proceedings of the Cambridge Philosophil Society. Vol. 60, pp. 667-674.

Bhuvanavijaya, R., Prasad, R. V., Mallikarjuna, B. and Beg, O. A. (2014): Natural convective heat transfer flow of a non-Newtonian second grade fluid past an isothermal sphere, Computational Thermal Science, Vol. 6(5), pp. 451-460:

Cebeci, T. and Bradshaw, P. (1984): Physical and Computational Aspects of Convective Heat Transfer, Springer-Verlin publication.

Cheng, C.Y. (2009): Natural convection heat transfer from a horizontal isothermal elliptical cylinder with internal heat generation. International Communications in Heat and Mass Transfer, Vol. 36, pp. 346-350. http://dx.doi.org/10.1016/j.icheatmasstransfer.2009.01.004

Fosdick, R.L. and Rajagopal, K.R. (1979): Anomalous features in the model of second order fluids, Arch. Rational. Mech. Anal., Vol. 70, pp. 145-152. http://dx.doi.org/10.1007/BF00250351

Gireesha, B .J., Chamkha, A. J., Manjunatha, S. and Bagewadi, C. S. (2013): Mixed convective flow of a dusty fluid over a vertical stretching sheet with non-uniform heat source/sink and radiation. Int. J of Numerical Method for heat and Fluid Flow, Vol. 23 (4), pp. 598-612: http://dx.doi.org/10.1108/09615531311323764,

Hayat, T., Abbas, Z., Sajid, M. and Asghar, S. (2007): The influence of thermal radiation on MHD flow of a second grade fluid. International Journal of Heat and Mass Transfer, Vol. 50, pp. 931-941. http://dx.doi.org/10.1016/j.ijheatmasstransfer.2006.08.014

Hayat, T. and Qasim, M. (2011): Radiation and magnetic field effects on the unsteady mixed convection flow of a second grade fluid over a vertical stretching sheet. International Journal For Numerical Methods in Fluids, Vol. 66, pp. 820-832. http://dx.doi.org/10.1002/fld.2285

Hussain, S. and Hussein, A. (2010): Numerical investigation of natural convection phenomena in a uniformly heated circular cylinder immersed in square enclosure filled with air at different vertical locations, International Communications in Heat and Mass Transfer, Vol. 37 (8), pp: 1115-1126.

Hussain, S. and Hussein, A. (2011): Natural convection heat transfer in a differentially heated square enclosure with a heat generating-conducting circular cylinder at different diagonal locations , $6^{\text {th }}$ International Advanced Technologies Symposium Proceedings (IATS'11), 16-18 May, Elazı̆g , Turkey, pp: 13 -19.

Hussein, A. (2013): Computational analysis of natural convection in a parallelogrammic cavity with a hot concentric circular cylinder moving at different vertical locations, International Communications in Heat and Mass Transfer, Vol. 46, pp : 126-133.

Mahmoud, M.A.E.H. (2008): Slip effects on flow and heat transfer of a non-Newtonian fluid on a stretching surface with thermal radiation. International Journal of Chemical Reactor Engineering, Vol. 6, pp. 1-20. http://dx.doi.org/10.2202/1542-6580.1792

Merkin, J. H. (1977): Free convection boundary layers on circular cylinders of elliptic cross section, Journal of Heat Transfer, Vol. 99, pp. 453-457: 
Mohammed, H. A. and Salman, Y. K. (2007): Experimental investigation of mixed convection heat transfer for thermally developing flow in a horizontal circular cylinder. Applied Thermal Engineering, Vol. 27, pp.15221533. http://dx.doi.org/10.1016/j.applthermaleng.2006.09.023

Molla, M. M., Hossain, M. A. and Goral, R. S. R. (2005): Natural convection flow from an isothermal horizontal circular cylinder with temperature dependent viscosity. Heat and Mass Transfer, Vol. 41, pp. 594598. http://dx.doi.org/10.1007/s00231-004-0576-7

Mukhopadhyay, S., Ranjan, P. D., Bhattacharyya, K. and Layek, G. C. (2012): Forced convective flow and heat transfer over a porous plate in a Darcy-Forchheimer porous medium in presence of radiation. Meccanica, Vol. 47, pp. 153-161: http://dx.doi.org/10.1007/s11012-011-9423-3

Na, T. Y. (1979): Computational Methods in Engineering Boundary Value Problems. Academic Press, Newyork.

Nadeem, S., Rehman, A., Lee, C., and Lee, J. (2012): Boundary layer flow of Second grade fluid in a cylinder with heat transfer. Mathematical Problems in Engineering, Article ID 640289, 13 pages. http://dx.doi.org/10.1155/2012/640289

Prasad, V. R., Rao, A. S. and Beg, O. A. (2013): Flow and heat transfer of Casson fluid from a horizontal circular cylinder with partial slip in non-Darcy porous medium. Applied \& Computational Mathematics, Vol. 2, Issue. 2, pp. 1-12. http://dx.doi.org/10.4172/2168-9679.1000127

Prasenjit, D., Abhijit, S. and Kumar, D. A. (2015): Prediction of unsteady mixed convection over circular cylinder in the presence of nanofluid-A comparative study of ANN and GEP. Journal of Naval Architecture and Marine Engineering, Vol. 12. Pp. 57-71: http://dx.doi.org/10.3329/jname.v12i1.21812.

Rashad, A. M. (2014): Effects of radiation and variable viscosity on unsteady MHD flow of a rotating fluid from stretching surface in porous medium, Vol. 22, pp. 134-142: http://dx.doi.org/10.1016/j.joems.2013.05.008.

Rashad, A. M, Mallikarjuna, B., Chamkha, A. J. and Raju, S. H. (2016): Thermophoresis effect on heat and mass transfer from a rotating cone in a porous medium with thermal radiation, Afrika Matematika, Article in Press, http://dx.doi.org/10.1007/s13370-016-0421-4

Rivlin, R. S. and Ericksen, J. L. (1995): Stress deformation relations for isotropic materials. J. Rat. Mech. Anal. Vol. 4, pp. 323-425. http://dx.doi.org/10.1007/978-1-4612-2416-7_61

Seddeek, M.A. (2006): Finite element method for the effect of various injection parameters on heat transfer for a power law non-Newtonian fluid over continues stretched surface with thermal radiation. Computation Material Science, Vol. 37 pp.624-627. http://dx.doi.org/10.1016/j.commatsci.2006.01.002

Siddheshwar, P. G. and Mahabaleswar, U. S. (2005): Effect of radiation and heat source on MHD flow of a viscoelastic liquid and heat transfer over a stretching sheet. International Journal of nonlinear mechanics, Vol. 40, pp. 807-820. http://dx.doi.org/10.1016/j.ijnonlinmec.2004.04.006

Srinivasacharya, D., Mallikarjuna, B. and Bhuvanavijaya, R. (2015): Radiation effect on mixed convection over a vertical wavy surface in Darcy porous medium with variable properties. Journal of Applied Science and Engineering, Vol. 18(3), pp. 265-274: http://dx.doi.org/10.6180/jase.2015.18.3.07

Tai, B. C. and Char, M. I. (2010): Soret and Dufour effects on free convection flow of non-Newtonian fluids along a vertical plate embedded in a porous medium with thermal radiation. International Communications in Heat and Mass Transfer, Vol. 37, pp. 480-483. http://dx.doi.org/10.1016/j.icheatmasstransfer.2009.12.017

Tham, L., Roslinda, N. and Pop, I. (2014): Mixed convection flow from a horizontal circular cylinder embedded in a porous medium filled by a nanofluid: Buongiorno-Darcy model. International Journal of Thermal Sciences, Vol. 84, pp. 21-33: http://dx.doi.org/10.1016/j.ijthermalsci.2014.04.020

Vasu, B., Prasad, V. R., Reddy, N. B. and Beg. O. A. (2010): Computational analysis of free convection heat and mass transfer from an isothermal horizontal circular cylinder in a micropolar fluid with Soret/Dufour effects. Proceedings of $37^{\text {th }}$ National $\& 4^{\text {th }}$ International conference on Fluid Mechanics and Fluid Power, Dec16-18, IIT Madras, India.

Yih, K. A. (2000): Effect of blowing/suction on MHD natural convection over horizontal cylinder: UWT or UHF, Acta Mechanicca, Vol. 144, pp. 17-27. 